EUROPEAN JOURNAL OF PURE AND APPLIED MATHEMATICS

Vol. 14, No. 4, 2021, 1184-1199

ISSN 1307-5543 - ejpam.com

Published by New York Business Global

\title{
Analytical Study for Certain Ordinary Differential Equations with Variable Coefficients via $G_{\alpha}$-Transform
}

Patarawadee Prasertsang $^{1}$, Supaknaree Sattaso ${ }^{1, *}, K^{2}$ amsing Nonlaopon ${ }^{2}$, Hwajoon $\mathrm{Kim}^{3}$

1 Department of General Science, Kasetsart University, Chalermphrakiat Sakon Nakhon Province Campus, Sakon Nakhon 47000, Thailand

${ }^{2}$ Department of Mathematics, Faculty of Science, Khon Kaen University, Khon Kaen 40002, Thailand

3 Department of IT Engineering, Kyungdong University, Yangju, Gyeonggi, Korea

\begin{abstract}
G_{\alpha}$-transform, which is a comprehensive and essential form of Laplace-type integral transforms, has both advantages and limitations. The purpose of this study is to consider the applicable range of $G_{\alpha}$-transform in finding solutions of ordinary differential equations with variable coefficients. Finally, several examples are given to demonstrate the effectiveness of these results.
\end{abstract}

2020 Mathematics Subject Classifications: 34A25, 34A26, 44A05

Key Words and Phrases: Laplace transform, Sumudu transform, Elzaki transform, $G_{\alpha}$-transform, Ordinary differential equation

\section{Introduction}

The differential equations have played a central role in every aspect of applied mathematics for a very long time, and their importance has increased further with the advent of computers. Several mathematical methods have been applied by various researchers in various fields of science and engineering to obtain the analytical solutions of differential equations, which appeared in the literature $[26,34,36]$. To solve the differential equations, the integral transforms were extensively used. The Laplace transform is one of many integral transforms in applied mathematics and is often used to solve differential equations.

The Laplace transform reduces a linear differential equation to an algebraic equation, which can then be solved using algebra's formal rules. After that, the differential equation can then be solved by applying the inverse Laplace transform [33]. The Laplace transform is beneficial for finding the solution of the diffusion equation in transient flow $[8,35,43]$. In

*Corresponding author.

DOI: https://doi.org/10.29020/nybg.ejpam.v14i4.4066

Email addresses: patarawadee.s@ku.th (P. Prasertsang), supaknaree.s@ku.th (S. Sattaso), nkamsi@kku.ac.th (K. Nonlaopon), cellmath@gmail.com (Hj. Kim) 
addition, many researchers mainly had paid attention to study for theory and applications of Laplace transform, see [9-11, 21, 41] for more details.

The Laplace transform is a well-known fact that it converts a function $f$ of a real variable $t$ to a function $F$ of a complex variable $s$, which is defined by

$$
F(s)=\mathcal{L}\{f(t)\}=\int_{0}^{\infty} e^{-s t} f(t) d t .
$$

In addition, if $f(t)$ is a piecewise continuous on $[0, \infty)$ and has an exponential order $k$, then the Laplace transform $F(s)=\mathcal{L}\{f(t)\}$ exists for $s>k$.

For $s=1 / u$, the Laplace transform $\mathcal{L}\{f(t)\}$ can be rewritten as

$$
\mathcal{L}\{f(t)\}=\int_{0}^{\infty} e^{-t / u} f(t) d t .
$$

In the last two decades, many integral transforms in the class of Laplace-typed integral transform are introduced, such as Sumudu transform, Elzaki transform, natural transform, Aboodh transform, Mohand transform, $G_{\alpha}$-transform, HY-transform, and Kamal transform. These transforms have been used for solving different types of integral equations, ordinary differential equations, partial differential equations, and fractional differential equations, see $[1,3,15,22,37,42,45]$ for more details.

Since the Laplace transform is not suitable for solving some differential equations, in 1993, G. Watugala [44] introduced a new transform, named Sumudu transform, and shown that Sumudu transform has fascinating properties, making it easy to visualize and apply it for finding the solution of ordinary differential equations in control engineering problems. Thus, the Sumudu transform is an ideal transform for control engineering and applied mathematics.

In 2010, H. Eltayeb and A. Kilicman [14] introduced some relationships between Sumudu transform and Laplace transform. They showed that the solution which is given by Laplace transform into a complex domain and given by Sumudu transform into a real domain. Thus, this leads them to consider that if the solution exists by Sumudu transform, then the solution also exists by Laplace transform. Moreover, they showed a strong relationship between Sumudu transform and other integral transforms, see A. Kilicman et al.[13].

Many researchers applied Sumudu transform to solve the system of dynamic equations, partial differential equations with variable coefficient, a semi-infinite string, an integrodifferential equation, the fractional neutron transport equation, see $[2,4-6,12,20,23-$ $25,27,28]$ for more details.

The Sumudu transform converts a function $f$ of a real variable $t$ to a function of a complex variable $u$, which is defined by

$$
S\{f(t)\}=\frac{1}{u} \int_{0}^{\infty} e^{-t / u} f(t) d t .
$$

In addition, if $f(t)$ is a piecewise continuous on $[0, \infty)$ and has an exponential order $k$, then the Sumudu transform $S\{f(t)\}$ exists for $u<1 / k$. 
Elzaki transform is the modified version of Laplace transform and Sumudu transform, which was first introduced by T.M. Elzaki [16] in 2011. Elzaki transform was then presented when Sumudu transform failed to solve some differential equations with variable coefficients [19]. T.M. Elzaki et al. [17, 18] showed that Elzaki transform provides a method for analyzing ordinary differential equations such as linear dynamic systems equation, signals-delay differential equation, and the renewal equation in statistics.

The Elzaki transform converts a function $f$ of a real variable $t$ to a function of a complex variable $u$, which is defined by

$$
E\{f(t)\}=u \int_{0}^{\infty} e^{-t / u} f(t) d t
$$

In particular, if $f(t)$ is a piecewise continuous on $t \geq 0$ and has an exponential order $k$, then the Elzaki transform $E\{f(t)\}$ exists for $u<1 / k$.

Recently, Hj. Kim [29] introduced the intrinsic structure and some properties of $G_{\alpha^{-}}$ transform, which is defined by

$$
F(u)=G_{\alpha}\{f(t)\}=u^{\alpha} \int_{0}^{\infty} e^{-t / u} f(t) d t,
$$

where $\alpha \in \mathbb{Z}$ and $u$ is a complex variable. The $G_{\alpha}$-transform can be applied directly to any situation by choosing $\alpha$ appropriately.

In addition, if $f(t)$ is a piecewise continuous on $t \geq 0$ and has an exponential order $k$, then the $G_{\alpha}$-transform $G_{\alpha}\{f(t)\}$ exists for $u<1 / k$.

The $G_{\alpha}$-transform is a Laplace-type integral transform can be reduced to the Laplace transform, Sumudu transform, and Elzaki transform for $\alpha=0,-1,1$, respectively.

Moreover, we know that the Laplace transform has a strong point in the transforms of derivatives. If we set $\alpha=-2$, then we obtain a simple tool for transforms of integral, which can be rewritten as

$$
G_{-2}\{f(t)\}=\frac{1}{u^{2}} \int_{0}^{\infty} e^{-t / u} f(t) d t
$$

see [30]. Further, Hj. Kim [31] also solved Laguerre's equation by the $G_{-2}$-transform.

In 2019, S. Sattaso et al. [39] studied the properties of $G_{\alpha}$-transform and presented an example that cannot be solved by the Sumudu and Elzaki transforms, but it can be solved by the $G_{\alpha}$-transform.

Furthermore, $\mathrm{Hj}$. Kim et al. [38] considered an application of $G_{\alpha}$-transform in partial differential equations by using the $n$-th partial derivatives, and $\mathrm{Hj}$. Kim [7, 32] also considered a proof concerning the Laplace transform of the $n$-th derivative of any order by mathematical induction and considered a variant of $G_{\alpha}$-transform represented by a logarithmic function. The connection of this transform to the convolutional neural network can be found in [40].

In this paper, we give some conditions of certain ordinary differential equations that can be solved by $G_{\alpha}$-transform. Furthermore, we include examples to demonstrate the effectiveness of these results. 


\section{Preliminaries}

In this section, we give some basic properties of the $G_{\alpha}$-transform, which would appear in this study quite frequently. The proofs of the following properties are given in [29, 39].

Lemma 1. [29] ( $G_{\alpha}$-transform of derivatives) If $f(t), f^{\prime}(t), \ldots, f^{(m-1)}(t)$ are continuous and $f^{(m)}(t)$ is a piecewise continuous function on $[0, \infty)$ for $m \in \mathbb{N} \cup\{0\}$ and has an exponential order $k$ for $u<1 / k$, then the following properties hold:

(i) $G_{\alpha}\left\{f^{\prime}(t)\right\}=\frac{F(u)}{u}-u^{\alpha} f(0)$;

(ii) $G_{\alpha}\left\{f^{\prime \prime}(t)\right\}=\frac{F(u)}{u^{2}}-u^{\alpha-1} f(0)-u^{\alpha} f^{\prime}(0)$;

(iii) $G_{\alpha}\left\{f^{(m)}(t)\right\}=\frac{F(u)}{u^{m}}-\sum_{k=0}^{m-1} u^{\alpha-m+(k+1)} f^{(k)}(0)$,

where $F(u)=G_{\alpha}\{f(t)\}$.

Lemma 2. [39] ( $G_{\alpha}$-transform of multiplication by power of $\left.t\right)$ If $f(t)$ is a piecewise continuous function on $[0, \infty)$ and has an exponential order $k$ for $u<1 / k$, then the following properties hold:

(i) $G_{\alpha}\{t f(t)\}=u^{2} F^{\prime}(u)-\alpha u F(u)$;

(ii) $G_{\alpha}\left\{t^{2} f(t)\right\}=u^{4} F^{\prime \prime}(u)-2(\alpha-1) u^{3} F^{\prime}(u)+(\alpha-1) \alpha u^{2} F(u)$;

(iii) $G_{\alpha}\left\{t^{n} f(t)\right\}=u^{2 n} F^{(n)}(u)-\left(\begin{array}{c}n \\ 1\end{array}\right)(\alpha-(n-1)) u^{2 n-1} F^{(n-1)}$

$$
\begin{aligned}
& +\cdots-\left(\begin{array}{c}
n \\
n-1
\end{array}\right)(\alpha-(n-1))(\alpha-(n-2)) \cdots(\alpha-1) u^{n+1} F^{\prime}(u) \\
& +(\alpha-(n-1))(\alpha-(n-2)) \cdots \alpha u^{n} F(u),
\end{aligned}
$$

where $F(u)=G_{\alpha}\{f(t)\}$.

Lemma 3. [39] If $f^{(m)}(t)$ is a piecewise continuous function on $[0, \infty)$ for $m \in \mathbb{N} \cup\{0\}$ and has an exponential order $k$ for $u<1 / k$, then

$$
\begin{aligned}
G_{\alpha}\left\{t^{n} f^{(m)}(t)\right\}= & u^{2 n} \frac{d^{n} G_{\alpha}\left\{f^{(m)}(t)\right\}}{d u^{n}}-\left(\begin{array}{c}
n \\
1
\end{array}\right)[\alpha-(n-1)] u^{2 n-1} \frac{d^{n-1} G_{\alpha}\left\{f^{(m)}(t)\right\}}{d u^{n-1}} \\
& +\cdots-\left(\begin{array}{c}
n \\
n-1
\end{array}\right)[\alpha-(n-1)][\alpha-(n-2)] \cdots(\alpha-1) u^{n+1} \frac{d G_{\alpha}\left\{f^{(m)}(t)\right\}}{d u} \\
& +[\alpha-(n-1)][\alpha-(n-2)] \cdots \alpha u^{n} G_{\alpha}\left\{f^{(m)}(t)\right\} .
\end{aligned}
$$

Lemma 4. [29] If $f(t)=t^{n}$ for $n \in \mathbb{N} \cup\{0\}$, then

$$
G_{\alpha}\left\{t^{n}\right\}=n ! u^{n+\alpha+1} .
$$


Remark 1. By using Lemma 3, substituting $n=1,2$, and 3 in (1) and derivatives, after some simplification, we obtain

(i) $G_{\alpha}\left\{t f^{(m)}(t)\right\}=\frac{F^{\prime}(u)}{u^{m-2}}-(m+\alpha) \frac{F(u)}{u^{m-1}}-\sum_{k=0}^{m-1}(1+k-m) u^{2+k+\alpha-m} f^{(k)}(0)$;

(ii) $G_{\alpha}\left\{t^{2} f^{(m)}(t)\right\}=\frac{F^{\prime \prime}(u)}{u^{m-4}}-2(m+\alpha-1) \frac{F^{\prime}(u)}{u^{m-3}}+[m(m+1)+2(\alpha-1) m+(\alpha-1) \alpha]$

$$
\begin{aligned}
& \times \frac{F(u)}{u^{m-2}}-\sum_{k=0}^{m-1}[(\alpha-m+k+1)(\alpha-m+k)-2(\alpha-1)(\alpha-m+k+1) \\
& +(\alpha-1) \alpha] u^{3+k+\alpha-m} f^{(k)}(0)
\end{aligned}
$$

(iii) $G_{\alpha}\left\{t^{3} f^{(m)}(t)\right\}=\frac{F^{\prime \prime \prime}(u)}{u^{m-6}}-3(m+\alpha-2) \frac{F^{\prime \prime}(u)}{u^{m-5}}+[3 m(m+1)+6(\alpha-2) m$

$$
\begin{aligned}
& +3(\alpha-2)(\alpha-1)] \frac{F^{\prime}(u)}{u^{m-4}}-[m(m+1)(m+2)+3(\alpha-2) m(m+1) \\
& +3(\alpha-2)(\alpha-1) m+(\alpha-2)(\alpha-1) \alpha] \frac{F(u)}{u^{m-3}} \\
& -\sum_{k=0}^{m-1}[(\alpha-m+k+1)(\alpha-m+k)(\alpha-m+k-1) \\
& -3(\alpha-2)(\alpha-m+k+1)(\alpha-m+k)+3(\alpha-2)(\alpha-1)(\alpha-m+k+1) \\
& -(\alpha-2)(\alpha-1) \alpha] u^{4+k+\alpha-m} f^{(k)}(0),
\end{aligned}
$$

where $F(u)=G_{\alpha}\{f(t)\}$.

\section{Main Results}

In this section, we show some conditions of certain ordinary differential equations to ensure that those ordinary differential equations can be solved by $G_{\alpha}$-transform.

Theorem 1. Consider the $m$-th order ordinary differential equation of the form

$$
\begin{aligned}
& \left(a_{m} t^{2}+b_{m} t+c_{m}\right) y^{(m)}(t)+\left(a_{m-1} t^{2}+b_{m-1} t+c_{m-1}\right) y^{(m-1)}(t) \\
& +\cdots+\left(a_{0} t^{2}+b_{0} t+c_{0}\right) y(t)=g(t),
\end{aligned}
$$

where $a_{j}, b_{j}, c_{j}$ are constants, $j=0,1,2, \ldots, m$ and $g(t)$ is an unknown function. The $G_{\alpha}$-transform is a suitable method for solving (2), if the following conditions are satisfies

$$
\begin{array}{r}
c_{m}=b_{m}=c_{m-1}=(\alpha-1) \alpha a_{0}=2(\alpha-1) a_{0}=0, \\
{[2+2(\alpha-1)+(\alpha-1) \alpha] a_{1}-\alpha b_{0}=b_{i-1}-2(\alpha+i-1) a_{i}=0}
\end{array}
$$

for $i=1,2,3, \ldots, m$, and

$$
[i(i+1)+2(\alpha-1) i+(\alpha-1) \alpha] a_{i}-(i+\alpha-1) b_{i-1}+c_{i-2}=0
$$

for $i=2,3,4, \ldots, m$. 
Proof. By using Remark 1(1-2) and taking $G_{\alpha}$-transform of both sides to (2), we obtain

$$
\begin{aligned}
& {\left[\frac{a_{m}}{u^{m-4}}+\frac{a_{m-1}}{u^{m-5}}+\cdots+\frac{a_{1}}{u^{-3}}+\frac{a_{0}}{u^{-4}}\right] F^{\prime \prime}(u)+\left[-2(\alpha+m-1) \frac{a_{m}}{u^{m-3}}-2(\alpha+m-2) \frac{a_{m-1}}{u^{m-4}}\right.} \\
& \left.-\cdots-2 \alpha \frac{a_{1}}{u^{-2}}-2(\alpha-1) \frac{a_{0}}{u^{-3}}+\frac{b_{m}}{u^{m-2}}+\frac{b_{m-1}}{u^{m-3}}+\cdots+\frac{b_{1}}{u^{-1}}+\frac{b_{0}}{u^{-2}}\right] F^{\prime}(u) \\
& +\left[(m(m+1)+2(\alpha-1) m+(\alpha-1) \alpha) \frac{a_{m}}{u^{m-2}}+((m-1) m+2(\alpha-1)(m-1)\right. \\
& +(\alpha-1) \alpha) \frac{a_{m-1}}{u^{m-3}}+\cdots+(2+2(\alpha-1)+(\alpha-1) \alpha) \frac{a_{1}}{u^{-1}}+(\alpha-1) \alpha \frac{a_{0}}{u^{-2}}-(\alpha+m) \frac{b_{m}}{u^{m-1}} \\
& \left.-(\alpha+m-1) \frac{b_{m-1}}{u^{m-2}}-\cdots-(\alpha+1) b_{1}-\alpha \frac{b_{0}}{u^{-1}}+\frac{c_{m}}{u^{m}}+\frac{c_{m-1}}{u^{m-1}}+\cdots+\frac{c_{1}}{u}+c_{0}\right] F(u) \\
& =G_{\alpha}\{g(t)\}-q(u),
\end{aligned}
$$

where $q(u)$ be contained in some expressions that are started by summation and do not influence the proof steps.

If the $G_{\alpha}$-transform is suitable method for solving (2), then the coefficient of $F(u)$ and $F^{\prime}(u)$ in (3) should be equal to zero. Thus, if the coefficient of $F(u)=0$, then

$$
\begin{aligned}
u^{m} & \rightarrow c_{m}=0 ; \\
u^{m-1} & \rightarrow c_{m-1}-(m+\alpha) b_{m}=0 ; \\
u^{m-2} & \rightarrow c_{m-2}-(m+\alpha-1) b_{m-1}+(m(m+1)+2(\alpha-1) m+(\alpha-1) \alpha) a_{m}=0 ; \\
& \vdots \\
u^{0} & \rightarrow c_{0}-(\alpha+1) b_{1}+(6+4(\alpha-1)+(\alpha-1) \alpha) a_{2}=0 ; \\
u^{-1} & \rightarrow-\alpha b_{0}+(2+2(\alpha-1)+(\alpha-1) \alpha) a_{1}=0 ; \\
u^{-2} & \rightarrow(\alpha-1) \alpha a_{0}=0 .
\end{aligned}
$$

And if the coefficient of $F^{\prime}(u)=0$, then

$$
\begin{aligned}
u^{m-2} & \rightarrow b_{m}=0 \\
u^{m-3} & \rightarrow b_{m-1}-2(m+\alpha-1) a_{m}=0 \\
u^{m-4} & \rightarrow b_{m-2}-2(m+\alpha-2) a_{m-1}=0 \\
\quad & \\
u^{-1} & \rightarrow b_{1}-2(\alpha+1) a_{2}=0 \\
u^{-2} & \rightarrow b_{0}-2 \alpha a_{1}=0 \\
u^{-3} & \rightarrow 2(\alpha-1) a_{0}=0 .
\end{aligned}
$$

In general, we can show that

$$
c_{m}=b_{m}=c_{m-1}=(\alpha-1) \alpha a_{0}=2(\alpha-1) a_{0}=0,
$$




$$
[2+2(\alpha-1)+(\alpha-1) \alpha] a_{1}-\alpha b_{0}=b_{i-1}-2(\alpha+i-1) a_{i}=0
$$

for $i=1,2,3, \ldots, m$, and

$$
[i(i+1)+2(\alpha-1) i+(\alpha-1) \alpha] a_{i}-(i+\alpha-1) b_{i-1}+c_{i-2}=0
$$

for $i=2,3,4, \ldots, m$. This completes the proof.

Remark 2. From Theorem 1, if $g(t)=0$, we can just set the coefficient of $F(u)$ equal to zero to reduce conditions. Therefore, the $G_{\alpha}$-transform is a suitable method for solving equation (2), if

$$
c_{m}=c_{m-1}-(m+\alpha) b_{m}=[2+2(\alpha-1)+(\alpha-1) \alpha] a_{1}-\alpha b_{0}=(\alpha-1) \alpha a_{0}=0,
$$

and

$$
[i(i+1)+2(\alpha-1) i+(\alpha-1) \alpha] a_{i}-(i+\alpha-1) b_{i-1}+c_{i-2}=0
$$

for $i=2,3,4, \ldots, m$.

Theorem 2. Consider the $m$-th order ordinary differential equation of the form

$$
\begin{aligned}
\left(a_{m} t^{3}+b_{m} t^{2}+c_{m} t+d_{m}\right) y^{(m)}(t) & +\left(a_{m-1} t^{3}+b_{m-1} t^{2}+c_{m-1} t+d_{m-1}\right) y^{(m-1)}(t) \\
& +\cdots+\left(a_{0} t^{3}+b_{0} t^{2}+c_{0} t+d_{0}\right) y(t)=g(t),
\end{aligned}
$$

where $a_{j}, b_{j}, c_{j}, d_{j}$ are constants, $j=0,1,2, \ldots, m$ and $g(t)$ is an unknown function. The $G_{\alpha^{-}}$-transform is a suitable method for solving (4), if the following conditions are satisfies

$$
\begin{array}{r}
d_{m}=c_{m}=b_{m}=d_{m-1}=c_{m-1}=d_{m-2}=0, \\
(\alpha-2)(\alpha-1) \alpha a_{0}=3(\alpha-2)(\alpha-1) a_{0}=3(\alpha-2) a_{0}=0, \\
\alpha c_{0}-[2+2(\alpha-1)+(\alpha-1) \alpha] b_{1} \\
+[24+18(\alpha-2)+6(\alpha-2)(\alpha-1)+(\alpha-2)(\alpha-1) \alpha] a_{2}=0, \\
(\alpha-1) \alpha b_{0}-[6+6(\alpha-2)+3(\alpha-2)(\alpha-1)+(\alpha-2)(\alpha-1) \alpha] a_{1}=0, \\
2(\alpha-1) b_{0}-[6+6(\alpha-2)+3(\alpha-2)(\alpha-1)] a_{1}=0, \\
d_{i-3}-(\alpha+i-2) c_{i-2}+[(i-1) i+2(\alpha-1)(i-1)+(\alpha-1) \alpha] b_{i-1}
\end{array}
$$

for $i=3,4,5, \ldots, m$,

$$
c_{i-2}-2(\alpha+i-2) b_{i-1}+[3 i(i+1)+6(\alpha-2) i+3(\alpha-2)(\alpha-1)] a_{i}=0
$$

for $i=2,3,4, \ldots, m$, and $b_{i-1}-3(\alpha+i-2) a_{i}=0$ for $i=1,2,3, \ldots, m$.

Proof. By using Remark 1 and taking $G_{\alpha}$-transform of both sides to (4), we obtain

$$
\left[\frac{a_{m}}{u^{m-6}}+\frac{a_{m-1}}{u^{m-7}}+\cdots+\frac{a_{1}}{u^{-5}}+\frac{a_{0}}{u^{-6}}\right] F^{\prime \prime \prime}(u)+\left[-3(m+\alpha-2) \frac{a_{m}}{u^{m-5}}-3(m+\alpha-3) \frac{a_{m-1}}{u^{m-6}}\right.
$$




$$
\begin{aligned}
& \left.-\cdots-3(\alpha-1) \frac{a_{1}}{u^{-4}}-3(\alpha-2) \frac{a_{0}}{u^{-5}}+\frac{b_{m}}{u^{m-4}}+\frac{b_{m-1}}{u^{m-5}}+\cdots+\frac{b_{1}}{u^{-3}}+\frac{b_{0}}{u^{-4}}\right] F^{\prime \prime}(u) \\
& +\left[(3 m(m+1)+6(\alpha-2) m+3(\alpha-2)(\alpha-1)) \frac{a_{m}}{u^{m-4}}+(3(m-1) m+6(\alpha-2)(m-1)\right. \\
& +3(\alpha-2)(\alpha-1)) \frac{a_{m-1}}{u^{m-5}}+\cdots+(6+6(\alpha-2)+3(\alpha-2)(\alpha-1)) \frac{a_{1}}{u^{-3}}+3(\alpha-2)(\alpha-1) \\
& \times \frac{a_{0}}{u^{-4}}-2(\alpha+m-1) \frac{b_{m}}{u^{m-3}}-2(\alpha+m-2) \frac{b_{m-1}}{u^{m-4}}-\cdots-2 \alpha \frac{b_{1}}{u^{-2}}-2(\alpha-1) \frac{b_{0}}{u^{-3}}+\frac{c_{m}}{u^{m-2}} \\
& \left.+\frac{c_{m-1}}{u^{m-3}}+\cdots+\frac{c_{1}}{u^{-1}}+\frac{c_{0}}{u^{-2}}\right] F^{\prime}(u)+[-(m(m+1)(m+2)+3(\alpha-2) m(m+1) \\
& +3(\alpha-2)(\alpha-1) m+(\alpha-2)(\alpha-1) \alpha) \frac{a_{m}}{u^{m-3}}-((m-1) m(m+1)+3(\alpha-2)(m-1) m \\
& +3(\alpha-2)(\alpha-1)(m-1)+(\alpha-2)(\alpha-1) \alpha) \frac{a_{m-1}}{u^{m-4}}-\cdots-(6+6(\alpha-2) \\
& +3(\alpha-2)(\alpha-1)+(\alpha-2)(\alpha-1) \alpha) \frac{a_{1}}{u^{-2}}-(\alpha-2)(\alpha-1) \alpha \frac{a_{0}}{u^{-3}}-(m(m+1) \\
& +2(\alpha-1) m+(\alpha-1) \alpha) \frac{b_{m}}{u^{m-2}}+((m-1) m+2(\alpha-1)(m-1)+(\alpha-1) \alpha) \frac{b_{m-1}}{u^{m-3}} \\
& +\cdots+(2+2(\alpha-1)+(\alpha-1) \alpha) \frac{b_{1}}{u^{-1}}+(\alpha-1) \alpha \frac{b_{0}}{u^{-2}}-(m+\alpha) \frac{c_{m}}{u^{m-1}} \\
& \left.+(m+\alpha-1) \frac{c_{m-1}}{u^{m-2}}-\cdots-(\alpha+1) \frac{c_{1}}{u^{0}}-\alpha \frac{c_{0}}{u^{-1}}+\frac{d_{m}}{u^{m}}+\frac{d_{m-1}}{u^{m-1}}+\cdots+\frac{d_{1}}{u^{1}}+\frac{d_{0}}{u^{0}}\right] F(u) \\
& +G_{\alpha}\{g(t)\}-r(u),
\end{aligned}
$$

where $r(u)$ be contained in some expressions that are started by summation and do not influence the proof steps.

By using the previous results, which similar to the Theorem 1, we know that the coefficients of $F(u), F^{\prime}(u)$ and $F^{\prime \prime}(u)$ should be equal to zero, by the same process as Theorem 1, we can show that

$$
\begin{aligned}
d_{m}=c_{m}=b_{m}=d_{m-1}=c_{m-1}=d_{m-2} & =0, \\
(\alpha-2)(\alpha-1) \alpha a_{0}=3(\alpha-2)(\alpha-1) a_{0}=3(\alpha-2) a_{0} & =0, \\
\alpha c_{0}-[2+2(\alpha-1)+(\alpha-1) \alpha] b_{1} & \\
+[24+18(\alpha-2)+6(\alpha-2)(\alpha-1)+(\alpha-2)(\alpha-1) \alpha] a_{2} & =0, \\
(\alpha-1) \alpha b_{0}-[6+6(\alpha-2)+3(\alpha-2)(\alpha-1)+(\alpha-2)(\alpha-1) \alpha] a_{1} & =0, \\
2(\alpha-1) b_{0}-[6+6(\alpha-2)+3(\alpha-2)(\alpha-1)] a_{1} & =0, \\
d_{i-3}-(\alpha+i-2) c_{i-2}+[(i-1) i+2(\alpha-1)(i-1)+(\alpha-1) \alpha] b_{i-1} & \\
-[i(i+1)(i+2)+3(\alpha-2) i(i+1)+3(\alpha-2)(\alpha-1) i+(\alpha-2)(\alpha-1) \alpha] a_{i} & =0
\end{aligned}
$$

for $i=3,4,5, \ldots, m$,

$$
c_{i-2}-2(\alpha+i-2) b_{i-1}+[3 i(i+1)+6(\alpha-2) i+3(\alpha-2)(\alpha-1)] a_{i}=0
$$

for $i=2,3,4, \ldots, m$, and $b_{i-1}-3(\alpha+i-2) a_{i}=0$ for $i=1,2,3, \ldots, m$. The proof is completed. 
Remark 3. From Theorem 2, if $g(t)=0$, we can just set the coefficient of $F(u)$ equal to zero and $F^{\prime}(u)$ equal to zero to reduce conditions. Therefore, the $G_{\alpha}$-transform is a suitable method for solving equation (4), if

$$
\begin{aligned}
d_{m}=c_{m}=d_{m-1}=c_{m-1}-2(\alpha+m-1) b_{m} & =0, \\
d_{m-2}-(\alpha+m-1) c_{m-1}+[m(m+1)+2(\alpha-1) m+(\alpha-1) \alpha] b_{m} & =0, \\
(\alpha-2)(\alpha-1) \alpha a_{0}=3(\alpha-2)(\alpha-1) a_{0} & =0, \\
\alpha c_{0}-[2+2(\alpha-1)+(\alpha-1) \alpha] b_{1}+ & \\
{[24+18(\alpha-2)+6(\alpha-2)(\alpha-1)+(\alpha-2)(\alpha-1) \alpha] a_{2} } & =0, \\
(\alpha-1) \alpha b_{0}-[6+6(\alpha-2)+3(\alpha-2)(\alpha-1)+(\alpha-2)(\alpha-1) \alpha] a_{1} & =0, \\
2(\alpha-1) b_{0}-[6+6(\alpha-2)+3(\alpha-2)(\alpha-1)] a_{1} & =0, \\
d_{i-3}-(\alpha+i-2) c_{i-2}+[(i-1) i+2(\alpha-1)(i-1)+(\alpha-1) \alpha] b_{i-1} & \\
-[i(i+1)(i+2)+3(\alpha-2) i(i+1)+3(\alpha-2)(\alpha-1) i+(\alpha-2)(\alpha-1) \alpha] a_{i} & =0
\end{aligned}
$$

for $i=3,4,5, \ldots, m$, and

$$
c_{i-2}-2(\alpha+i-2) b_{i-1}+[3 i(i+1)+6(\alpha-2) i+3(\alpha-2)(\alpha-1)] a_{i}=0
$$

for $i=2,3,4, \ldots, m$.

\section{Examples}

In this section, we show the usage of $G_{\alpha}$-transform for solving the ordinary differential equations with variable coefficients that according to Theorem 1 and Theorem 2 via some examples.

Example 1. Consider the ordinary differential equation with variable coefficients of the form

$$
t^{2} y^{\prime \prime}(t)+4 t y^{\prime}(t)+2 y(t)=t^{3} .
$$

From (2) and (5), we have

$$
a_{2}=1, \quad b_{1}=4, \quad c_{0}=2, \quad a_{0}=a_{1}=0, \quad b_{0}=b_{2}=0, \quad c_{1}=c_{2}=0,
$$

and we define $\alpha=1$ to satisfy with the conditions of Theorem 1, so using the $G_{1}$-transform leads to find the solution of (5). By applying the $G_{1}$-transform to (5) and using Lemma 3, we obtain

$$
\begin{aligned}
G_{1}\left\{t^{2} y^{\prime \prime}(t)\right\}+G_{1}\left\{4 t y^{\prime}(t)\right\}+G_{1}\{2 y(t)\} & =G_{1}\left\{t^{3}\right\} \\
u^{2} F^{\prime \prime}(u)-4 u F^{\prime}(u)+6 F(u)+4 u F^{\prime}(u)-8 F(u)+2 F(u) & =6 u^{5} \\
F^{\prime \prime}(u) & =6 u^{3} .
\end{aligned}
$$

Then, we have

$$
F(u)=\frac{3}{10} u^{5}+c_{1} u+c_{2},
$$


where $c_{1}$ and $c_{2}$ are constants. Letting $c_{1}=c_{2}=0$, we get $F(u)=\frac{3}{10} u^{5}$. By using Lemma 4 and the inverse $G_{1}$-transform, thus the inverse of $u^{5}$ is $\frac{t^{3}}{6}$, we obtain $y(t)=\frac{1}{20} t^{3}$ as a solution of (5). It is not difficult to show that $y(t)=\frac{1}{20} t^{3}$ satisfies (5).

The next example will show that if the conditions do not satisfy Theorem 1, then it is not suitable to solve by this method as the following.

Example 2. Consider the Legendre differential equation of the form

$$
\left(1-t^{2}\right) y^{\prime \prime}(t)-2 t y^{\prime}(t)=t .
$$

From (2) and (6), we have

$$
a_{2}=-1, \quad c_{2}=1, \quad b_{1}=-2, \quad a_{0}=a_{1}=0, \quad b_{0}=b_{2}=0, \quad c_{0}=c_{1}=0,
$$

and with respect to the conditions in Theorem 1, $c_{2}$ should be equal to 0 , while $c_{2}$ is equal to 1 . Therefore, the conditions of Theorem 1 are not satisfied. If we take $G_{\alpha}$-transform both sides of (6), we obtain

$$
\begin{aligned}
G_{\alpha}\left\{\left(1-t^{2}\right) y^{\prime \prime}(t)\right\}-G_{\alpha}\left\{2 t y^{\prime}(t)\right\} & =G_{\alpha}\{t\} \\
-u^{2} F^{\prime \prime}(u)+2 \alpha u F^{\prime}(u)+\left((\alpha-3) \alpha+\frac{1}{u^{2}}\right) F(u) & =u^{\alpha+2} .
\end{aligned}
$$

Observe that (6) changed into a second-order ordinary differential equation with variable coefficients. Thus, using $G_{\alpha}$-transform did not lead to finding the solution of (6).

Example 3. Consider the ordinary differential equation with variable coefficients of the form

$$
t^{2} y^{\prime \prime}(t)+2 t y^{\prime}(t)-2 y(t)=0
$$

From (2) and (7), we have

$$
a_{2}=1, \quad b_{1}=2, \quad c_{0}=-2, \quad a_{0}=a_{1}=0, \quad b_{0}=b_{2}=0, \quad c_{1}=c_{2}=0,
$$

and we define $\alpha=1$ to satisfy with the conditions of Remark 2, so using the $G_{1}$-transform leads to find the solution of (7). By applying the $G_{1}$-transform to (7) and using Lemma 3, we obtain

$$
\begin{aligned}
G_{1}\left\{t^{2} y^{\prime \prime}(t)\right\}+G_{1}\left\{2 t y^{\prime}(t)\right\}-G_{1}\{2 y(t)\} & =0 \\
u^{2} F^{\prime \prime}(u)-4 u F^{\prime}(u)+6 F(u)+2 u F^{\prime}(u)-2 F(u)-2 F(u)-2 F(u) & =0 \\
u^{2} F^{\prime \prime}(u)-2 u F^{\prime}(u) & =0 .
\end{aligned}
$$

Then, we have $\frac{F^{\prime \prime}(u)}{F^{\prime}(u)}=\frac{2}{u}$. By integration both sides, we obtain

$$
\ln F^{\prime}(u)=\ln c_{1} u^{2} \quad \text { or } \quad F^{\prime}(u)=c_{1} u^{2}
$$


and hence

$$
F(u)=\frac{c_{1}}{3} u^{3}+c_{2},
$$

where $c_{1}$ and $c_{2}$ are constants. Letting $c_{2}=0$, we get $F(u)=\frac{c_{1}}{3} u^{3}$. By using Lemma 4 and the inverse $G_{1}$-transform, thus the inverse of $u^{3}$ is $t$, we obtain $y(t)=\frac{c_{1}}{3} t$ as a solution of (7). It is not difficult to show that $y(t)=\frac{c_{1}}{3} t$ satisfies (7).

Example 4. Consider the ordinary differential equation with variable coefficients of the form

$$
t^{3} y^{\prime \prime \prime}(t)+9 t^{2} y^{\prime \prime}(t)+18 t y^{\prime}(t)+6 y(t)=t .
$$

From (4) and (8), we have

$a_{3}=1, \quad b_{2}=9, \quad c_{1}=8, \quad d_{0}=6, \quad a_{0}=a_{1}=a_{2}=0, \quad b_{0}=b_{1}=b_{3}=0, \quad c_{0}=c_{2}=c_{3}=0$, $d_{1}=d_{2}=d_{3}=0$,

and we define $\alpha=2$ to satisfy with the conditions of Theorem 2, so using the $G_{2}$-transform leads to find the solution of (8). By applying the $G_{2}$-transform to (8) and using Lemma 3, we obtain

$$
\begin{aligned}
G_{2}\left\{t^{3} y^{\prime \prime \prime}(t)\right\}+G_{2}\left\{9 t^{2} y^{\prime \prime}(t)\right\}+G_{2}\left\{18 t y^{\prime}(t)\right\}+G_{2}\{6 y(t)\} & =G_{2}\{t\} \\
u^{3} F^{\prime \prime \prime}(u)-9 u^{2} F^{\prime \prime}(u)+36 u F^{\prime}(u)-60 F(u)+9 u^{2} F^{\prime \prime}(u)-54 u F^{\prime}(u)+108 F(u) & \\
+18 u F^{\prime}(u)-54 F(u)+6 F(u) & =u^{4} .
\end{aligned}
$$

Then, we have $F^{\prime \prime \prime}(u)=u$. By integration both sides, we obtain

$$
F(u)=\frac{1}{24} u^{4}+\frac{c_{1}}{2} u^{2}+c_{2} u+c_{3},
$$

where $c_{1}, c_{2}$, and $c_{3}$ are constants. Letting $c_{1}=c_{2}=c_{3}=0$, we get $F(u)=\frac{1}{24} u^{4}$. By using Lemma 4 and the inverse $G_{2}$-transform, thus the inverse of $u^{4}$ is $t$, we obtain $y(t)=\frac{1}{24} t$ as a solution of (8).

The next example will show that if the conditions do not satisfy Theorem 2 , then it is not suitable to solve by this method as the following.

Example 5. Consider the ordinary differential equation with variable coefficients of the form

$$
\left(t^{3}+t\right) y^{\prime \prime \prime}(t)+6 t^{2} y^{\prime \prime}(t)+6 t y^{\prime}(t)=t^{2} .
$$

From (4) and (9), we have

$$
\begin{aligned}
& a_{3}=1, \quad c_{3}=1, \quad b_{2}=6, \quad c_{1}=6, \quad a_{0}=a_{1}=a_{2}=0, \quad b_{0}=b_{1}=b_{3}=0, \quad c_{0}=c_{2}=0, \\
& d_{0}=d_{1}=d_{2}=d_{3}=0
\end{aligned}
$$


and with respect to the conditions in Theorem 2, $c_{3}$ should be equal to 0 , while $c_{3}$ is equal to 1. Therefore, the conditions of Theorem 2 are not satisfied. If we take $G_{\alpha}$-transform both sides of (9), we obtain

$$
\begin{gathered}
G_{\alpha}\left\{\left(t^{3}+t\right) y^{\prime \prime \prime}(t)\right\}+G_{\alpha}\left\{6 t^{2} y^{\prime \prime}(t)\right\}+G_{\alpha}\left\{6 t y^{\prime}(t)\right\}=G_{\alpha}\left\{t^{2}\right\} \\
u^{3} F^{\prime \prime \prime}(u)-[3+3(\alpha-2)] u^{2} F^{\prime \prime}(u)+[18-18(\alpha-2)+3(\alpha-2)(\alpha-1) \\
\left.-12(\alpha-1)+\frac{1}{u^{2}}\right] u F^{\prime}(u)-[24+36(\alpha-2)+9(\alpha-2)(\alpha-1) \\
\left.+(\alpha-2)(\alpha-1) \alpha+(\alpha+3) \frac{1}{u^{2}}-24(\alpha-1)+6(\alpha+1)\right] F(u)=2 u^{\alpha+3} .
\end{gathered}
$$

Observe that (9) changed into a third order ordinary differential equation with variable coefficients. Thus, by using $G_{\alpha}$-transform did not lead to find the solution of (9).

Example 6. Consider the ordinary differential equation with variable coefficients of the form

$$
t^{3} y^{\prime \prime \prime}(t)+4 t^{2} y^{\prime \prime}(t)-2 t y^{\prime}(t)-4 y(t)=0 .
$$

From (4) and (10), we have

$$
\begin{aligned}
& a_{3}=1, \quad b_{2}=4, \quad c_{1}=-2, \quad d_{0}=-4, \quad a_{0}=a_{1}=a_{2}=0, \quad b_{0}=b_{1}=b_{3}=0, \\
& c_{0}=c_{2}=c_{3}=0, \quad d_{1}=d_{2}=d_{3}=0,
\end{aligned}
$$

and we define $\alpha=1$ to satisfy with the conditions of Remark 3, so using the $G_{1}$-transform leads to find the solution of (10). By applying the $G_{1}$-transform to (10) and using Lemma 3, we obtain

$$
\begin{aligned}
G_{1}\left\{t^{3} y^{\prime \prime \prime}(t)\right\}+G_{1}\left\{4 t^{2} y^{\prime \prime}(t)\right\}-G_{1}\left\{2 t y^{\prime}(t)\right\}-G_{1}\{4 y(t)\} & =0 \\
u^{3} F^{\prime \prime \prime}(u)-9 u^{2} F^{\prime \prime}(u)+36 u F^{\prime}(u)-60 F(u)+3 u^{2} F^{\prime \prime}(u)-18 u F^{\prime}(u)+36 F(u) & \\
+4 u^{2} F^{\prime \prime}(u)-16 u F^{\prime}(u)+24 F(u)-2 u F^{\prime}(u)+2 F(u)+2 F(u)-4 F(u) & =0 .
\end{aligned}
$$

Then, we have $\frac{F^{\prime \prime \prime}(u)}{F^{\prime \prime}(u)}=\frac{2}{u}$. By integration both sides, we obtain

$$
\ln F^{\prime \prime}(u)=\ln c_{1} u^{2} \quad \text { or } \quad F^{\prime \prime}(u)=c_{1} u^{2},
$$

and hence

$$
F(u)=\frac{c_{1}}{12} u^{4}+c_{2} u+c_{3},
$$

where $c_{1}, c_{2}$, and $c_{3}$ are constants. Letting $c_{2}=c_{3}=0$, we get $F(u)=\frac{c_{1}}{12} u^{4}$. By using Lemma 4 and the inverse $G_{1}$-transform, thus the inverse of $u^{4}$ is $\frac{t^{2}}{2}$, we obtain $y(t)=\frac{c_{1}}{24} t^{2}$ as a solution of (10). 
Remark 4. We can see that Example 1, 3, and 6 can be solved by $G_{1}$-transform, and Example 4 can be solved by $G_{2}$-transform, it is clear that Sumudu transform cannot be solved for these ordinary differential equations.

Remark 5. If we choose the suitable value for $\alpha$ and the problem is consistent with the conditions of Theorem 1 or Theorem 2, then we can easily find the solution of the ordinary differential equation. But if the problem is not consistent with the conditions of Theorem 1 or Theorem 2, it will be difficult to find the solution of the ordinary differential equation.

\section{Conclusions}

We obtained some conditions of certain ordinary differential equations to ensure that it can be solved by $G_{\alpha}$-transform. In this regard, we observed that $G_{\alpha}$-transform more appropriate than other Laplace-typed integral transforms to solve the ordinary differential equations with variable coefficients by choosing the suitable value for $\alpha$.

\section{Acknowledgements}

This research was supported by Faculty of Sciences and Engineering, Kasetsart University, Chalermprakiat Sakon Nakhon Province Campus, Thailand.

\section{References}

[1] K.S. Aboodh. The new integral transform Aboodh transform. Global Journal of Pure and Applied Mathematics, 9(1):35-43, 2013.

[2] H.A. Agwa, F.M. Ali, and A. Kilicman. A new integral transform on time scales and its applications. Advances in Difference Equations, 60:1-14, 2012.

[3] Z. Al-Zhour, N. Al-Mutairi, F. Alrawajeh, and R. Alkhasawneh. New theoretical results and applications on conformable fractional Natural transform. Ain Shams Engineering Journal, 2(1):927-933, 2020.

[4] M.A. Asiru. Further properties of the Sumudu transform and its applications. International Journal of Mathematical Education in Science and Technology, 33(3):441-449, 2002.

[5] A. Atangana and A. Akgul. Can transfer function and Bode diagram be obtained from Sumudu transform. Alexandria Engineering Journal, 59:1971-1984, 2020.

[6] H. Bulut, H.M. Baskonus, and S. Tuluce. The solutions of partial differential equations with variable coefficient by Sumudu transform method. In K Girardi, editor, 9th International Conference on Mathematical Problems in Engineering; the American Institute of Physics., pages 91-95, New York, 2012. AIP Conference Proceedings. 
[7] Hj. Kim, S. Beak, and J. Rho. Variant of Laplace transform represented by a logarithmic function. International Journal of Difference Equations, 15(1):71-82, 2020.

[8] G.B. Davis. A Laplace transform technique for the analytical solution of a diffusionconvection equation over a finite domain. Applied Mathematical Modelling, 9:69-71, 1985.

[9] L. Debnath. Integral Transforms and their Applications. Taylor \& Francis Group, Milton Park, Oxfordshire, 1995.

[10] G. Doetsch. Guide to the Applications of Laplace Transforms. Van Nostrand Company, New York, USA, 1963.

[11] G. Doetsch. Introduction to the Theory and Application of the Laplace Transform. Springer-Verlag Berlin Heidelberg, New York, USA, 1970.

[12] A. Kilicman, H. Eltayeb, and R.P. Agarwal. On Sumudu transform and system of differential equations. Abstract and Applied Analysis, 11 pages, 2011.

[13] A. Kilicman, H. Eltayeb, and K.A.M. Atan. A note on the comparison between Laplace and Sumudu transforms. Iranian Mathematical Society, 37(1):131-141, 2011.

[14] H. Eltayeb and A. Kilicman. A note on the Sumudu transforms and differential equations. Applied Mathematical Sciences, 4(22):1089-1098, 2010.

[15] A.A. Alderremy, T.M. Elzaki, and M. Chamekh. New transform iterative method for solving some Klein-Gordon equations. Results in Physics, 10:655-659, 2018.

[16] T.M. Elzaki. On the connections between Laplace and Elzaki transforms. Advances in Theoretical and Applied Mathematics, 6(1):1-11, 2011.

[17] T.M. Elzaki and S.M. Elzaki. On the Elzaki transform and ordinary differential equation with variable coefficients. Advances in Theoretical and Applied Mathematics, 6(1):41-46, 2011.

[18] T.M. Elzaki, S.M. Elzaki, and E.A. Elnour. On some applications of new integral transform "Elzaki transform". Global Journal of Mathematical Sciences: Theory and Practical, 4(1):15-23, 2012.

[19] T.M. Elzaki, S.M. Elzaki, and E.M.A. Hilal. Elzaki and Sumudu transforms for solving some differential equations. Global Journal of Pure and Applied Mathematics, $8(2): 167-173,2012$.

[20] A.K. Golmankhaneh and T. Tunc. Sumudu transform in fractal calculus. Applied Mathematics and Computation, 350:386-401, 2019.

[21] A.C. Grove. An Introduction to the Laplace Transform and the Z-Transform. Prentice Hall, Hoboken, New Jersey, 1991. 
[22] S.A.P. Ahmadi, H. Hosseinzadeh, and A.Y. Cherati. A new integral transform for solving higher order linear ordinary differential equations. Nonlinear Dynamics and Systems Theory, 19(2):243-252, 2019.

[23] H. Jafari. A new general integral transform for solving fractional integral equation. Journal of Advanced Research Available, https://doi.org/10.1016/j.jare.2020.08.016, 2020 .

[24] A. Kadem. Solving the one-dimensional neutron transport equation using Chebyshev polynomials and the Sumudu transform. Analele Universitatii din Oradea, 12:153$171,2005$.

[25] A. Kadem and A. Kilicman. Note on transport equation and fractional Sumudu transform. Computers and Mathematics with Applications, 62:2995-3003, 2011.

[26] S. Chakraverty, N.R. Mahato, P. Karunakar, and T.D. Rao. Advanced Numerical and Semi-Analytical Methods for Differential Equations. John Wiley \& Sons, Hoboken, New Jersey, 2019.

[27] A. Kilicman and H.E. Gadain. On the applications of Laplace and Sumudu transforms. Journal of the Franklin Institute, 347(5):848-862, 2010.

[28] H. Eltayeb, A. Kilicman, and B. Fisher. A new integral transform and associated distributions. Integral Transforms and Special Functions, 21(5-6):367-379, 2010.

[29] Hj. Kim. The intrinsic structure and properties of Laplace-typed integral transforms. Mathematical Problems in Engineering, 8 pages, 2017.

[30] $\mathrm{Hj}$. Kim. On the form and properties of an integral transform with strength in integral transforms. Far East Journal of Mathematical Sciences, 102(11):2831-2844, 2017.

[31] Hj. Kim. The solution of Laguerre's equation by using $G$-transform. International Journal of Applied Engineering Research, 12(24):16083-16086, 2017.

[32] Hj. Kim. A proof with respect to Laplace transform of the $n$-th derivative by mathematical induction. Advances in Dynamical Systems and Applications, 15(1):29-33, 2020 .

[33] G.A. Korn and T.M. Korn. Mathematical Handbook for Scientists and Engineers (2nd ed.). McGraw-Hill Companies, Mineola, New York, 1968.

[34] N. Kumar and R. Kumar. Differential Equations and Integral Transforms. CBS Publishers and Distributors, Uttar Pradesh, India, 2016.

[35] E. Momoniat, R. McIntyre, and R. Ravindran. Numerical inversion of a Laplace transform solution of a diffusion equation with a mixed derivative term. Applied Mathematics and Computation, 209(2):222-229, 2009. 
[36] J.W. Miles. Integral Transforms in Applied Mathematics. Cambridge university press, Cambridge, England, 2008.

[37] M. Mohand and A. Mahgoub. The new integral transform "Mohand transform". Advances in Theoretical and Applied Mathematics, 12(2):113-120, 2017.

[38] Hj. Kim, S. Sattaso, K. Nonlaopon, and K. Kaewnimit. An application of generalized Laplace transform in PDEs. Advances in Dynamical Systems and Applications, $14(2): 257-265,2019$.

[39] S. Sattaso, K. Nonlaopon, and Hj. Kim. Further properties of Laplace-typed integral transforms. Dynamic Systems and Applications, 28(1):195-215, 2019.

[40] Y.H. Geum, A.K. Rathie, and Hj. Kim. Matrix expression of convolution and its generalized continuous form. Symmetry, 12(11):1791, 2020.

[41] J.L. Schiff. The Laplace Transform: Theory and Applications. Springer-Verlag Berlin Heidelberg, New York, USA, 1999.

[42] S. Aggarwal, N. Sharma, and R. Chauhan. Duality relations of Kamal transform with Laplace, Laplace-Carson, Aboodh, Sumudu, Elzaki, Mohand and Sawi transforms. SN Applied Sciences, 2(1):135, 2020.

[43] A. Tagliani and M. Milev. Laplace transform and finite difference methods for the Black-Scholes equation. Applied Mathematics and Computation, 220:649-658, 2013.

[44] G. Watugala. Sumudu transform: a new integral transform to solve differential equations and control engineering problems. Integrated Education, 24(1):35-43, 1993.

[45] S. Weerakoon. Application of Sumudu transform to partial differential equations. International Journal of Mathematical Education in Science and Technology, 25(2):277283, 1994. 